CARNETS DE Carnets de géographes

GÉOGRAPHES

\title{
Nostalgie et géographie
}

Entretien avec Philippe Gervais-Lambony

Pauline Guinard et Philippe Gervais-Lambony

\section{(2) OpenEdition}

Journals

Édition électronique

URL : http://journals.openedition.org/cdg/618

DOI : $10.4000 /$ cdg.618

ISSN : 2107-7266

Éditeur

UMR 245 - CESSMA

Référence électronique

Pauline Guinard et Philippe Gervais-Lambony, « Nostalgie et géographie », Carnets de géographes [En ligne], 9 | 2016, mis en ligne le 30 novembre 2016, consulté le 14 septembre 2020. URL : http:// journals.openedition.org/cdg/618

\section{(c) $(7)(8)$}

La revue Carnets de géographes est mise à disposition selon les termes de la Licence Creative Commons Attribution - Pas d'Utilisation Commerciale - Pas de Modification 4.0 International. 


\section{NOSTALGIE ET GEOGRAPHIE \\ Entretien avec Philippe Gervais-Lambony}

Réalisé par Pauline Guinard, le 7 janvier 2016.

Philippe Gervais-Lambony est Professeur de géographie à l'Université de Paris Nanterre, chercheur à I'UMR LAVUE, membre de l'Institut Universitaire de France et rédacteur en chef de la revue Justice Spatiale/Spatial Justice (jssj.org). Spécialiste des villes africaines et plus particulièrement sud-africaines, ses recherches portent sur la construction territoriale des identités, la justice spatiale et le droit à la ville. Adepte d'une approche qualitative qui accorde une attention privilégiée à la littérature, il s'intéresse depuis une quinzaine d'années à la nostalgie. C'est sur cette thématique que porte essentiellement cet entretien.

\section{A la recherche de la nostalgie}

Pauline Guinard (PG) : Pourquoi et comment en êtes-vous venu à travailler sur la nostalgie ?

Philippe Gervais-Lambony (PGL) : Je pense que cela date du début des années 2000. C'est certainement lié au fait de travailler en Afrique du Sud et même plus précisément d'abord à Johannesburg, puis dans ce qui s'appelait l'East Rand [Ekurhuleni aujourd'hui] ${ }^{1}$. Ce sont, en effet, des espaces qui ont connu des périodes considérées comme des périodes de gloire ou de grand succès mondial, puis des phases de dépression. Généralement, ce n'est pas tout l'espace urbain qui est concerné, mais certains espaces tel le centre-ville de Johannesburg. Dans les années 1990 en Afrique du Sud, il y avait un double regard sur le passé en général, et sur le passé du centre-ville de Johannesburg en particulier. D'un côté, il y avait ce discours qu'on percevait déjà (même peut-être plus qu'aujourd'hui) des habitants pour qui le centre-ville de Johannesburg représentait un âge d'or passé. Et ce souvenir pouvait d'ailleurs être partagé par les anciens habitants noirs de Johannesburg, qui racontaient que quand ils venaient dans le centre, quand ils étaient enfants, donc sous l'apartheid, ils étaient admiratifs de ce centre-ville qui leur semblait magnifique. De l'autre côté, il y avait une autre forme de regard sur le passé, liée aux destructions de quartiers orchestrées par l'apartheid (Sophiatown à Johannesburg,

\footnotetext{
${ }^{1}$ Les propos entre crochets, de même que les notes, sont de P. Guinard.
} 
District 6 au Cap, etc.) et aux déplacements forcés ${ }^{2}$ qui ont suivi ces destructions. Travaillant sur des questions d'identifications territoriales, liées donc à l'espace et aux mémoires, j'ai alors commencé à me demander : qu'est-ce que ce regard ambigu ? Qu'est-ce que ce sentiment ? Estce de la nostalgie ? Et consécutivement, peut-on étudier la nostalgie en géographie ?

Ce questionnement m'est apparu encore plus vivement quand j'ai commencé à travailler sur l'East Rand à la fin des années 1990, et ce d'autant plus qu'au même moment je lisais City of Quartz de Mike Davis (1997) et son très beau chapitre "Junkyard of dreams ${ }^{3}$ ». Dans le cas de I'East Rand, on avait affaire à un vieil espace minier, puis industriel, en complet effondrement économique, mais qui, à un moment donné, avait été porteur de l'idée qu'il constituait une sorte de centre mondial. Ce discours était particulièrement fort au moment même où l'on reconstruisait la métropole de l'East Rand en la rebaptisant et en la redéfinissant territorialement. Je crois que c'est de cette façon que j'en suis venu à travailler sur la nostalgie.

PG : La notion de nostalgie était-elle utilisée par les gens que vous interrogiez ou est-ce vous qui avez qualifié de nostalgie ce « regard ambigu sur le passé »?

PGL : C'est venu plus tard, par hasard, sur le terrain. Un de mes proches interlocuteurs, qui est un élu local et avec lequel je travaillais jusque-là sur des questions de politiques locales, me dit un jour: "Ah! Il s'est passé quelque chose dans le quartier où j'ai grandi ». Et je lui demande de me raconter. II me dit : "Non, je vais t'emmener voir l'endroit ». II s'agissait de Comet, une ancienne cité minière sur laquelle j'ai, depuis, travaillé et publié [voir par exemple : GervaisLambony, 2012]. II me fait visiter son quartier et me présente des gens qui étaient des amis d'enfance et ces derniers commencent à me parler, à me raconter: " On faisait du vélo ensemble. Le week-end on faisait ça. On était agressé par les enfants des familles blanches qui habitaient juste à côté. Etc. » Et donc, je leur demande : "Vous êtes vraiment dans un discours sur le passé. Est-ce que vous regrettez ce passé ? ". Ils me répondent : " C'est embêtant comme question parce qu'évidemment ce passé c'était l'apartheid, et puis après on est devenu des militants politiques ». Et mon interlocuteur principal en particulier, il ne pouvait pas dire : « Je regrette ce temps-là. " Ils n'utilisaient donc pas la notion de nostalgie, mais ce qu'ils évoquaient était tellement lié aux souvenirs d'un lieu et, en même temps, aux souvenirs d'un temps, qu'on était exactement dans un sentiment nostalgique.

A partir de là, la question était : pourquoi ce sentiment ? Ce qui supposait de se demander, premièrement, comment gérer des bons souvenirs personnels d'une époque " mauvaise ", pour le dire simplement, ce sur quoi a d'ailleurs travaillé par la suite Jacob Dlamini dans Native Nostalgia (2010); deuxièmement, pourquoi ce sentiment-là est apparu à ce moment-là (début des années 2000), en Afrique du Sud, et qu'est-ce que cela signifiait quant à l'évolution de la société sud-africaine; et troisièmement, qu'est-ce que cela signifiait plus généralement dans

\footnotetext{
${ }^{2}$ La mise en place de l'apartheid (1948) et la systématisation de la séparation des populations selon leur « race » a conduit à des démolitions de quartiers, ainsi qu'au déplacement de leurs habitants vers des espaces généralement périphériques et conçus par le régime pour être « racialement » homogènes.

${ }^{3}$ Le chapitre est intitulé « Le dépotoir de rêves » dans la version française.
} 
une réflexion sur l'attachement aux lieux. En ce qui me concerne, c'est aussi pour répondre à ces questions que je mobilise aussi des œuvres littéraires qui en traitent.

PG : Et donc votre intérêt pour la littérature est né à ce moment-là, parce que vous y avez trouvé des réponses, des manières d'approcher ce sentiment ?

PGL : J'ai toujours travaillé avec de la littérature! Depuis mes études de géographie, depuis mon DEA (Diplôme d'Etudes Approfondi, ex-Master 2 de recherche), qui portait sur l'image de la ville dans le roman africain. Et il se trouve que beaucoup d'auteurs sud-africains traitent de la nostalgie. Directement, en utilisant le terme.

PG : Vous pensez à Ivan Vladislavic?

PGL : I. Vladislavic a une grande influence sur ce sujet. C'est un auteur majeur. Mais, dans les auteurs plus anciens, celui qui me fascine depuis longtemps c'est Herman Charles Bosman. II traite de nostalgie à une époque de modernisation intense pour Johannesburg, les années 1940 surtout, à une période de destructions, de constructions, de destructions créatrices si vous voulez. Et il s'interroge sur ces destructions, qui se font avec le soutien des autorités locales, et l'effacement des traces du passé. De même dans les pièces de théâtre d'Athol Fugard, vous trouvez beaucoup de choses sur la mémoire et son importance. Donc il y a énormément de choses sur la mémoire dans la littérature sud-africaine. Pour beaucoup d'auteurs contemporains, s'interroger sur la mémoire suppose de se demander comment on traite la question de l'apartheid et des souvenirs de l'apartheid. Mais, déjà, dans des œuvres plus anciennes de la littérature sud-africaine cette question est centrale. Si vous lisez Down $2^{\text {nd }}$ Avenue d'Es'kia Mphahlele (1985 [1959]), vous trouvez cela : à la fois l'expression d'une forte nostalgie de l'enfance de l'auteur dans le quartier Sophiatown et une critique acerbe de l'oppression dans une société raciste. La littérature a donc simplement renforcé mon intérêt pour la nostalgie. Et cela n'est pas seulement vrai pour les auteurs sud-africains.

PG : Alors là, vous pensez à qui ? Proust ?

PGL : Je pense à Proust. Et en même temps... Le processus est peut-être le suivant. On a une approche qui intègre la littérature, cela conduit - du fait du terrain - à une thématique qui est fortement géographique, parce que je ne vois pas comment on pourrait dire que la nostalgie n'est pas liée à une relation à l'espace, à une relation au lieu. A partir de là, on découvre tout un champ de recherche qui traite de la nostalgie dans d'autres domaines ou espaces. II y a beaucoup d'écrits notamment sur les villes d'Europe de l'Est, c'est-à-dire des situations urbaines au fond assez comparables à celles sud-africaines, parce qu'elles correspondent à des situations avec des ruptures très fortes et une gestion du passé difficile. Qu'est-ce qu'on fait des monuments? Qu'est-ce qu'on fait des traces de ce passé dans l'espace ? Et puis, en littérature on s'aperçoit - enfin on s'en aperçoit parce qu'on n'y avait pas pensé avant - que c'est un thème majeur, qui traverse toute la littérature $d u X X^{e}$ siècle, en commençant bien sûr par Baudelaire, puis Benjamin sur Baudelaire, etc. Voilà, c'est un effet boule-de-neige. 


\section{Nostalgie, nostalgies}

PG : Revenons à la notion de nostalgie. Vous avez dit qu'elle était liée à un temps passé, à un espace passé, mais comment la définiriez-vous ? Est-ce, pour vous, une émotion ou un sentiment ? Est-elle collective ou individuelle?

PGL : Au départ, j'ai essayé de construire des catégories, de définir des formes différentes de nostalgie, ce qu'on peut d'ailleurs ramener à ce que je disais sur la différence entre la nostalgie pour le passé glorieux d'un espace ou la nostalgie pour un espace qui a été détruit. J'avais proposé une catégorisation en trois grands champs :

1. Le premier, je l'avais appelé un peu ambitieusement la nostalgie pré-moderne. Cela correspondait au fond à l'exil, c'est-à-dire à la première définition de la nostalgie comme arrachement à un lieu et regret de ce lieu. En ce qui concerne l'exil, vous pouvez remonter très loin, puisque c'est Virgile le grand auteur de cette nostalgie-là, à la suite bien sûr d'Homère. Et cela fonctionne à toutes les échelles : la sortie d'un territoire national par exemple, mais aussi l'arrachement à un lieu comme cela fût le cas en Afrique du Sud pour les habitants de quartiers détruits.

2. Ensuite, j'avais proposé de parler d'une nostalgie caractéristique de la modernité. C'est celle de Baudelaire, celle du Cygne dans lequel il écrit ces vers si souvent cités : "la forme d'une ville / Change plus vite, hélas! que le cœur d'un mortel ». C'est ce qui marque le $X I X{ }^{e}$ siècle évidemment. Cette nostalgie n'est pas liée au déplacement de la personne, mais elle est liée à la destruction des lieux, à leur changement, à leur transformation II n'y a pas de déplacement mais changement de l'espace. Et d'une certaine manière, cette nostalgie est aussi travaillée par David Harvey [voir par exemple : Harvey, 1989] pour qui il s'agit d'étudier des réactions au changement de l'espace urbain, à la destruction créatrice capitaliste.

3. Et puis, troisième grande famille de nostalgie, qu'on pouvait appeler post-moderne. C'était l'idée que l'on constate aujourd'hui une production massive de représentations du passé que des auteurs, à l'instar d'Arjun Appadurai (2005 [1996]), définissent comme une caractéristique forte des sociétés post-coloniales, au sens où elles sont productrices d'une nostalgie de ce qu'on n'a pas connu. A. Appadurai lie cela au pouvoir de l'imagination, qui favoriserait le développement d'un ersatz de nostalgie au service d'un marketing spatial mondialisé. On n'a aucune difficulté à trouver des exemples de cela, surtout sur les terrains sur lesquels je travaille. A Johannesburg, le processus de production, notamment en périphérie, d'espaces qui sont inspirés d'un passé souvent toscan ou oriental est massif. C'est bien du passé, mais les gens ne l'ont jamais connu. Cela n'a aucun sens théoriquement. Et pourtant, cela correspond à une forme de marketing de la nostalgie. Par ailleurs, beaucoup de politiques patrimoniales peuvent être interprétées de cette façon, c'est-à-dire comme une production de patrimoine qui n'a pas grand chose à voir avec ce que les gens ont connu, avec ce dont ils ont fait l'expérience.

Donc, voilà, j'avais fait cette catégorisation là [voir notamment : Gervais-Lambony, 2003, 2012]. 
Puis, j'ai rencontré d'autres catégorisations dont celle de Stevlana Boym (2001) qui distingue nostalgie restaurative et nostalgie réflexive. La première porte à regretter le passé et à vouloir y retourner, à vouloir revenir en arrière. Pour S. Boym, cette nostalgie est dangereuse politiquement en tant qu'elle est une forme de conservatisme. C'est le retour en arrière. Et c'est aussi le nationalisme, ou du moins un des éléments du nationalisme. La seconde nostalgie, qui est réflexive, c'est presque le contraire. C'est l'idée que la nostalgie donne aussi le sens du caractère éphémère de toute chose, et notamment des civilisations et des espaces urbains. Cette nostalgie permet de relativiser et de penser les transformations, tout en se tournant vers le futur. Donc, une nostalgie qui est tournée vers le passé, l'autre qui permet de penser le futur et d'accepter la perte du présent.

A partir de là, pour moi la question était de savoir dans quelle mesure la nostalgie avait des dimensions spatiales afin de traiter la question en géographe. S. Boym dit que la nostalgie est une " historical emotion" (" émotion historique "). Mais, je me demande si ce n'est pas plutôt une "spatial emotion ", c'est-à-dire si on ne l'éprouve pas forcément la nostalgie dans une relation à l'espace. Cela dit, je ne pense que cela ait beaucoup de sens que d'essayer de distinguer le temps et l'espace : les deux sont liés. Le temps, c'est de l'espace. L'espace, c'est du temps. Et chez Proust, c'est terriblement lié : il y a une indissociabilité complète du temps et de l'espace. D. Harvey (1989) montre aussi bien cela : on se remémore le temps passé à travers des mémoires d'espaces, des souvenirs de lieux. Donc il y a quelque chose d'éminemment géographique. Pour moi, c'était très important de légitimer l'étude de la nostalgie comme approche géographique: la nostalgie me semble nécessairement concerner la géographie humaine, parce que je ne vois pas comment on peut traiter de l'humain sans traiter de ce qui est l'une de ses caractéristiques, à savoir éprouver des sentiments et des émotions.

Alors après, est-ce que c'est un sentiment ou une émotion ? Au fond, je me suis jamais posé la question, sauf en lisant l'appel à contribution de ce numéro spécial des Carnets de géographes. A la lecture de ce texte, il me semblait qu'il serait peut-être intéressant de faire une distinction entre, d'une part, l'émotion provoquée par un événement soudain (dans l'appel il était fait mention des émotions provoquées par les attentats de début 2015, et de tout ce que cela provoque comme effets qui sont effectivement pour une part spatiaux), et, d'autre part, quelque chose qui serait plutôt de l'ordre du sentiment, qui se déploierait sur une durée plus longue, telle la nostalgie qui se construit dans le temps. Mais que la nostalgie soit un sentiment ou une émotion, ce que j'aime dans le terme d'émotion c'est qu'il renvoie à une mise en mouvement, à de l'action. Et la nostalgie provoque de l'action, comme le dit S. Boym, dans un bon ou dans un mauvais sens mais elle provoque de l'action. Et cela a beaucoup de conséquences, notamment parce cela donne une dimension politique à toute cette réflexion.

PG : Avec la question du politique, on touche également à la dimension potentiellement collective de la nostalgie, non ?

PGL : Oui, tout à fait. La question de l'individuel et du collectif est un point important parce qu'il y a beaucoup de pièges à éviter à ce sujet. La nostalgie est un sentiment individuel, que tout le monde (avec plus ou moins d'acuité) peut éprouver ; la nostalgie de l'enfance ou de la jeunesse, et donc pour les lieux de l'enfance, est sa forme la plus classique. C'est un sentiment individuel 
qui, comme tout sentiment, a des effets sur les comportements individuels. Mais c'est aussi quelque chose de très manipulé ou utilisé - on peut être positif ou négatif - pour produire du collectif. Dans la construction des identités territoriales notamment, la référence au passé est toujours très forte.

Je reviens au quartier de Comet. Je m'étais donc demandé pourquoi à ce moment-là un sentiment nostalgique était apparu. En fait, il y avait une raison très précise : le quartier était menacé de destruction. C'est parce que les habitants l'avaient appris qu'ils avaient commencé à produire collectivement ce discours sur le passé du quartier, qu'ils avaient recontacté des anciens habitants qui en étaient partis et qui pouvaient avoir un rôle politique, qu'ils avaient fait appel à la presse, etc. Ils avaient même mobilisé des membres, en majorité blancs, de l'association de défense du patrimoine historique de la région du fait de la présence dans la quartier de bâtiments dessinés par des associés d'Herber Baker ${ }^{4}$. C'était donc un discours nostalgique, instrumentalisé de façon à pouvoir se tourner vers les autorités métropolitaines pour défendre le quartier, pour empêcher sa destruction et l'expulsion de ses habitants. Et d'ailleurs, cela a eu les effets escomptés puisque récemment, pour répondre à la protestation des habitants, la métropole a racheté le quartier aux investisseurs fonciers privés qui l'avaient eux-mêmes racheté à la compagnie minière. Donc c'est très clair : il y a eu manipulation. Mais ce n'est pas pour autant que le sentiment de nostalgie n'est pas partagé par les habitants. En tant que chercheur, on peut faire un choix dans ce type de situation: soit travailler sur la manipulation, soit travailler sur ce sentiment. A mon sens, l'instrumentalisation ne décrédibilise pas du tout le sentiment en lui-même. Pour autant, il ne faut pas tomber dans le piège de ne pas voir que c'est une instrumentalisation. Et cela vaut encore à toutes les échelles.

Si l'on revient aux attentats, aux tueries successives de 2015, c'est la même chose. Il s'agit aussi de réfléchir à comment ces événements sont utilisés, instrumentalisés, manipulés, ce qui ne retire rien à l'importance de s'interroger sur l'émotion suscitée par ces événements et aux conséquences sur nos espaces et nos vies. La question - qui n'est pas originale, ni spécifique à ces sujets - est alors de savoir comment s'articulent les deux échelles (individuelle et collective). L'un peut-il aller sans l'autre ? Le sentiment individuel naît-il d'une manipulation ou est-ce l'inverse ? Dans quel ordre se déroule ce processus et qu'est-ce que cela nous apprend ? D'où l'importance de la question de S. Boym qui permet de distinguer les "bons " et les " mauvais » usages de la mémoire.

PG: La question de l'usage suppose d'ailleurs d'aborder le sentiment ou l'émotion non seulement dans le sentir mais aussi dans les conséquences de ce ressenti.

PGL : Oui, qu'est-ce qu'on fait de ce sentiment et qui le fait ? Pour moi, cela a un lien très fort avec les question de justice spatiale, c'est-à-dire les questions de droit et même directement de droit à la ville. Savoir qui a le droit ou pas de produire un discours sur le passé, de produire un discours qui est éventuellement nostalgique. II y a des nostalgies interdites et des nostalgies autorisées par les autorités. A ce sujet, on pourrait prendre des cas extrêmes. II y a des nostalgies contre lesquelles il faut lutter: les nostalgies de violence, d'autoritarisme, etc. Par

\footnotetext{
${ }^{4}$ H. Baker (1862-1946), grand architecte de l'empire britannique, auteur de plusieurs bâtiments officiels d'Afrique du Sud (notamment Union Buildings à Pretoria) et principal architecte de New Delhi.
} 
contre, dans le cas de l'apartheid, c'est plus problématique quand on considère, non pas la nostalgie des promoteurs de l'apartheid, mais cette nostalgie individuelle des vécus sous l'apartheid. On ne peut pas l'étouffer ! Et on la voit apparaître dans des réunions de quartier, notamment à Comet, qui était une cité minière construite sous l'apartheid pour le régime de l'apartheid. Or, cela fait aussi partie du droit des habitants que d'exprimer un sentiment de nostalgie pour une période qui n'est vraiment pas positive. Cela ouvre tout un champ de recherche sur la participation citadine à la production des mémoires, qui est aussi une participation politique.

PG : Puisqu'on parle du lien entre nostalgie et (droit à la) ville, pensez-vous qu'il y ait une dimension proprement urbaine de la nostalgie ? Ou pour le dire autrement la nostalgie en ville est-elle spécifique?

PGL: S'il y a une spécificité de l'espace urbain - ce que l'on pourrait d'ailleurs discuter aujourd'hui-, c'est d'être changeant. II y a des cycles. On y détruit et on y construit. Et aujourd'hui, dans l'ensemble des villes du monde, on est dans un système d'économie capitaliste qui a besoin de cette construction/destruction. Et le rythme s'accélère. Alors je dirais que c'est aussi une caractéristique du citadin que de devoir être en mesure de vivre et supporter ce changement permanent qui en plus s'accélère. Cela suppose de gérer la nostalgie que ce changement permanent provoque, de gérer la mémoire. Je ne dirais surtout pas que les espaces ruraux ne changent pas, qu'ils ne sont pas affectés par des changements, mais je pense que les rythmes sont différents, que les natures du changement sont différentes. Donc oui, à mon avis oui, il y a une dimension à la fois urbaine et contemporaine à la nostalgie.

PG : Et sud-africaine?

PGL : Non, je ne crois pas. La nostalgie a des caractéristiques particulières en Afrique du Sud du fait de l'apartheid. Mais cela vaut pour l'ensemble des territoires qui ont connu des ruptures politiques très fortes, des passages de régimes autoritaires à autre chose. A ce titre, on pourrait essayer de distinguer des types d'espaces urbains : des villes plus ou moins anciennes, des villes dites des nouveaux mondes qui n'ont pas ou peu de profondeur historique. Nous avions écrit avec Sophie Didier, qui a beaucoup travaillé ces questions notamment à Los Angeles, et d'autres, un texte dans Vies Citadines (Dorier-Apprill et Gervais-Lambony, 2007) autour de l'idée des villes sans mémoire. Mais la nostalgie concerne l'urbain en général, ce sont ses modalités qui diffèrent d'une ville à l'autre et d'un temps à l'autre.

\section{PG : Est-ce que les villes sans mémoire sont sans nostalgie?}

PGL: Non, au contraire, puisqu'elles sont dans des processus de destruction souvent encore plus rapides, elles suscitent encore davantage de sentiments nostalgiques.

PG : Parce qu'il y a un besoin de mémoire plus important ?

PGL: Parce qu'il y a une présence du passé dans l'espace beaucoup moins importante et des ruptures spatiales beaucoup plus brutales. Si l'on est d'accord pour dire que le sentiment de nostalgie est lié à ces transformations de l'espace, cette différence là est essentielle. D'où l'importance des tomasons. 
PG : Parlons des tomasons. Cette figure étant importante dans votre travail, peut-être que vous pourriez nous expliquer ce qu'elle recouvre et quel est son lien à la nostalgie.

PGL : Oui, elle est importante, surtout quand on se demande comment le passé est présent dans l'espace. Il est présent par des discours portés sur l'espace et il est présent dans l'espace, matériellement, concrètement, par des objets urbains, des bâtiments, des monuments anciens, etc. C'est dans cette catégorie qu'il faut ranger les tomasons : ce sont des objets du passé toujours présents. Mais ensuite, on peut faire une seconde subdivision parmi ces objets du passé toujours présents: d'un côté, ceux qui sont patrimonialisés, produits, reconnus comme des objets du passé et éventuellement protégés et, d'un autre côté, ceux qui ne le sont pas, ceux qui sont des accidents, ceux qui sont des choses qui restent parce qu'on a oublié de les détruire. Ceux-là sont des tomason. Le tomason est une notion qui a été inventée par un artiste japonais, Genpei Akasegawa ${ }^{5}$ (2010 [1987]). Pour lui, une des caractéristiques des espaces métropolitains, c'est d'être parsemés d'objets incompréhensibles, uniques, qui sont des restes de passé oublié. II utilise le terme de tomason en référence à un joueur de baseball états-unien, arrivé au Japon dans les années 1980 et devenu totalement inefficace. Il n'est donc plus qu'une sorte de relique. On ne sait plus à quoi il sert! Henri Desbois ${ }^{6}$ a écrit des choses très intéressantes là-dessus parce qu'au Japon la question des tomasons a pris une certaine dimension et a été repris par des auteurs de science-fiction, notamment Gibson. Mais, I. Vladislavic, auteur sud-africain, a également repris cette notion pour parler de Johannesburg dans Portrait with keys :

The natural habitat of the tomason is the city street. [...] Tomasons thrive in the manmade world, in spaces that are constantly being remade and redesigned for other purposes, where the function of a thing that was useful and necessary may be swept away in a tide of change or washed off like a label. (2006, p. 163-164)

Dans ce passage, I. Vladislavic raconte comment, marchant dans la rue, il découvre une sorte de poteau qui ne sert à rien, mais qui a une forme particulière. II se demande ce que c'est que cette chose, à quoi elle pouvait bien servir, si cela faisait partie d'un ensemble plus grand, etc. Et il appelle cela un tomason. Et pour moi, c'est exactement ce que Michel de Certeau (1994 [1980]) appelle un revenant. C'est un objet oublié et cet objet provoque des sentiments, qui sont une interrogation sur le passé, qui sont éventuellement une nostalgie et qui sont, en même temps, une conscience de l'éphémère parce qu'on se dit que cet objet qui est oublié, il va finir par être repéré et supprimé. Ces objets sont essentiels aussi parce qu'ils n'entrent pas dans le système spatial et économique capitaliste, ils n’ont pas leur place dans la ville néolibérale et peuvent donc être une forme de résistance matérielle à sa complète réalisation.

\footnotetext{
${ }^{5}$ G. Akasegawa propose l'orthographe "thomasson » en référence au joueur de baseball Gary Thomasson, alors qu'I. Vladislavic utilise une version simplifiée du terme ("tomason »), qui est reprise par P. Gervais-Lambony dans ses textes et par nous-mêmes ici.

${ }^{6}$ Voir notamment son intervention lors du séminaire « Mémoire des lieux. La ville en images » du laboratoire Mosaïques de mars 2014 : http://www.lavue.cnrs.fr/2actualites/2seminaires/MOSAIQUES/ProgrammeMemoireLieuxVillelmage2014/Program meMemoireLieuxVillelmage032014.pdf
} 
Donc, d'un côté, il y a la patrimonialisation, de l'autre côté, les objets oubliés ou ce que j'appelle les tomasons. Cette partition a un lien fort avec les deux types de mémoire de Proust (volontaire, involontaire). On pourrait dire que la mémoire volontaire, c'est ce que propose le patrimoine, ce qu'on met en avant et que la mémoire involontaire, c'est ce que rend possible le tomason. Or, Proust dit que la mémoire volontaire n'est qu'une reconstruction qui ne permet pas de revivre les émotions passées. A l'inverse, la mémoire involontaire serait la seule manière de faire l'expérience d'une "reviviscence ". C'est pour cela qu'il est important d'avoir dans les villes ces revenants, ces tomasons. Et de Certeau ne dit rien d'autre dans son texte sur les " revenants de la ville » (1994 [1980]) dans lequel il oppose, d'un côté la patrimonialisation, la prise de contrôle de la mémoire par la patrimonialisation, et, de l'autre côté, ces objets accidentels qui ouvrent l'espace urbain sur le passé, qui ouvrent les habitants des villes sur le passé.

C'est pour cela que la notion de tomason est utile. Elle est en outre directement liée à des questions spatiales: on a une présence dans l'espace d'objets du passé, généralement petits. Mais pourquoi ne pourrait-on pas appliquer cela à d'autres choses que des objets ? Si l'on change d'échelle, on pourrait l'appliquer à des quartiers, des secteurs urbains, des villes comme par exemple d'anciennes villes minières ou industrielles, soit des espaces qui faisaient partie d'un système économique qui n'existe plus mais qui restent là en attendant de faire l'objet d'une phase de destruction ou de patrimonialisation. Ce type d'espaces existe dans tous les pays qui ont connu des phases d'industrialisation ou des changements de phases économiques, que ce soit en France, aux Etats-Unis ou en Afrique du Sud. Si on change d'échelle, la notion de tomason devient donc très riche pour les géographes parce que c'est une autre façon de définir les espaces et les problèmes posés par ces espaces. C'est dans ces cas-là, que l'on trouve à la fois des phénomènes d'instrumentalisation de la nostalgie pour défendre des territoires et des sentiments vécus très forts de nostalgie pour un espace mais aussi pour un système économique du passé et pour une période qui était plus heureuse parce qu'elle était économiquement plus dynamique. Et plus les cycles économiques sont rapides, plus ce type d'espaces se multiplie comme c'est le cas depuis une quarantaine d'années. Mais, dans le même temps, plus les politiques de contrôle et de marchandisation de l'espace sont efficaces plus les objets et espaces urbains inutiles et non-désirés sont efficacement effacés.

PG : Mais si un tomason peut être patrimonialisé, est-ce que l'inverse est vrai ? Est-ce qu'il y a des patrimoines qui perdent finalement leur fonction, leur sens, jusqu'à devenir des tomasons ?

PGL: Si je prends l'exemple des monuments et des statues d'un régime passé, et par exemple ceux du régime de l'apartheid, est-ce qu'ils deviennent des tomasons quand le régime change ? A ce titre, le cas de la statue de Rhodes au Cap est intéressant. Les étudiants de l'Université du Cap ont manifesté en 2015 pour demander la suppression de ce symbole colonial.

PG : Mais pourquoi ce retrait a été réclamé et obtenu maintenant, soit 20 ans après la fin de l'apartheid ? On en revient à des questions de temporalités. Alors que l'Afrique du Sud avait fait le choix de garder ces statues héritées de l'apartheid, aujourd'hui elles tombent.

PGL : Est-ce que c'est lié au sentiment de manque de transformation de l'Afrique du Sud ? On a cru un temps qu'on pouvait changer le pays sans changer les symboles qui le représentaient. Maintenant qu'il y a une forte insatisfaction face au manque de transformation, on passe à une 
nouvelle phase. Vous pouvez dire la même chose des changements de noms de rues. Le travail sur la toponymie est au fond très tardif en Afrique du Sud. Cela a commencé il y a seulement quelques années, ville par ville, espace par espace. II y a donc ces questions de monuments, mais il y a aussi ces questions de la dénomination des lieux hérités du passé.

PG : Serait-ce à lier à un changement des formes de nostalgie?

PGL : Une compétition des nostalgies plutôt, un conflit des nostalgies contradictoires qui se joue en Afrique du Sud entre les mémoires des différents groupes culturels: la mémoire conservatrice souvent éprouvée par des Sud-Africains "blancs ", qui sont eux-mêmes divers et qui ne sont d'ailleurs pas les seuls à ressentir ce type de nostalgie, la mémoire de l'esclavage, la mémoire de l'apartheid, les mémoires des différentes communautés sociales sud-africaines, etc. Il y avait à ce sujet un dessin extraordinaire de Zapiro sur le changement de nom de Prétoria devenue Tshwane (Figure 1).

Figure 1 : Le changement de nom de Prétoria vu par Zapiro, un conflit de nostalgies ?

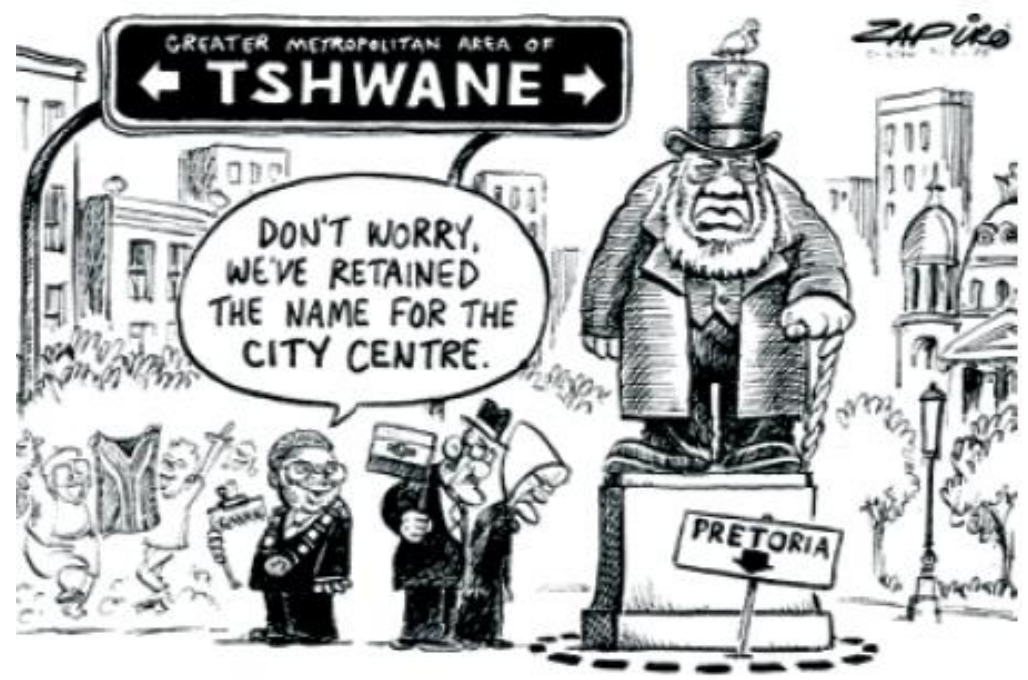

Source : Mail \& Guardian, 9 mars 2005.

On a bien là un conflit de mémoire. Mais est-ce qu'il y deux nostalgies : celle sur le passé de l'apartheid, mais aussi celle des populations noires, celle des opposants au régime pour l'époque assez simple - simple parce que claire, parce qu'on savait contre quoi on se battait de la lutte contre l'apartheid ? Et comment travailler ces questions en géographe?

\section{Nostalgies, littérature, chansons et subjectivités}

PG : Venons en précisément à la question des outils pour étudier la nostalgie en géographe. Vous nous avez dit faire notamment appel à la littérature, mais comment travaillez-vous la nostalgie à partir de la littérature?

PGL : On n'est pas obligé de mobiliser la littérature pour travailler sur ces questions. Il se trouve que c'est un support que j'affectionne, et notamment, assez paradoxalement, pour ses apports 
théoriques. Les cas de Proust, de Saint-Exupéry, de Camus ou de Cendrars sont à cet égard intéressants. Ce sont des auteurs qui, dans leur œuvre littéraire, tiennent un discours théorique sur le monde. Par exemple, je fais un usage largement théorique de La Recherche du temps perdu en utilisant les notions de mémoires volontaire et involontaire. En l'occurrence, je ne fais pas dans ce cas un usage de Proust différent de celui que je peux faire de Michel de Certeau ou d'Henri Lefebvre. C'est qu'il y a plusieurs manières d' « utiliser la littérature ». On peut l'utiliser en tant qu'elle est un discours sur un espace de référence, en tant qu'elle produit elle-même de l'espace, etc. II y a tout un champ de géo-littérature autour de cela. Mais, à mon sens, il existe une autre approche qui consiste à prendre au sérieux les apports théoriques d'auteurs qui sont aussi des écrivains, poètes ou romanciers.

Quand on travaille sur la nostalgie, il y a en effet tout un volet discours à analyser (ceux des habitants, des citadins, mais aussi ceux des autorités, des acteurs économiques, etc.) dans lequel on peut ranger la littérature et pas seulement la littérature. On peut y inclure la chanson, la musique, la peinture, etc. On sait bien à quel point, par exemple, la chanson, surtout populaire, est empreinte de discours sur la nostalgie, notamment en Afrique du Sud mais aussi ailleurs. Un exemple classique est celui de la chanson de Charles Trenet, Ménilmontant. Cette chanson est extrêmement claire sur le lien entre un lieu du passé et une personne, parce que si l'on écoute la chanson jusqu'à la fin, on s'aperçoit que la nostalgie de Trenet pour un lieu du passé est aussi une nostalgie pour sa mère. Le sentiment de nostalgie pour un espace ici n'a de sens que parce que cet espace est lié à la relation avec une personne : on regrette des lieux parce qu'on regrette des personnes avec qui on a vécu dans ces lieux, qu'on a connues dans ces lieux. C'est pour cela que je disais que la nostalgie est profondément humaine. En ce sens, l'étudier, c'est faire de la géographie humaine, voire humaniste.

Quant aux chanteurs sud-africains, il faudrait citer Hugh Mashekela qui a chanté les mineurs, les souvenirs des mineurs et des mines, les chanteurs de kwaito et de hip-hop sud-africain (je connais moins bien cette musique, mais elle comprend de nombreuses références au passé), Miriam Makeba qui a chanté Sophiatown très peu de temps après sa destruction ou encore Brenda Fassie qui est un personnage vraiment intéressant, parce qu'elle était une chanteuse populaire, qui ne se revendiquait pas du tout politiquement, en tout cas jusqu'à la fin de l'apartheid. A la fin de l'apartheid, elle a fait une chanson sur Mandela, qui évoque le passé, l'oppression, etc. C'est intéressant aussi parce que maintenant elle est patrimonialisée. Elle a sa statue dans le quartier de Newtown à Johannesburg (Figure 2). Elle y est patrimonialisée comme une femme noire et libre, qui à sa manière contestait l'oppression de l'apartheid parce qu'elle revendiquait le droit à être cette femme libre, avec ses aventures sexuelles, etc. 
Figure 2: La statue de Brenda Fassie (1964-2004) à Newtown (Johannesburg), une patrimonialisation (nostalgique ?) d'une chanteuse populaire

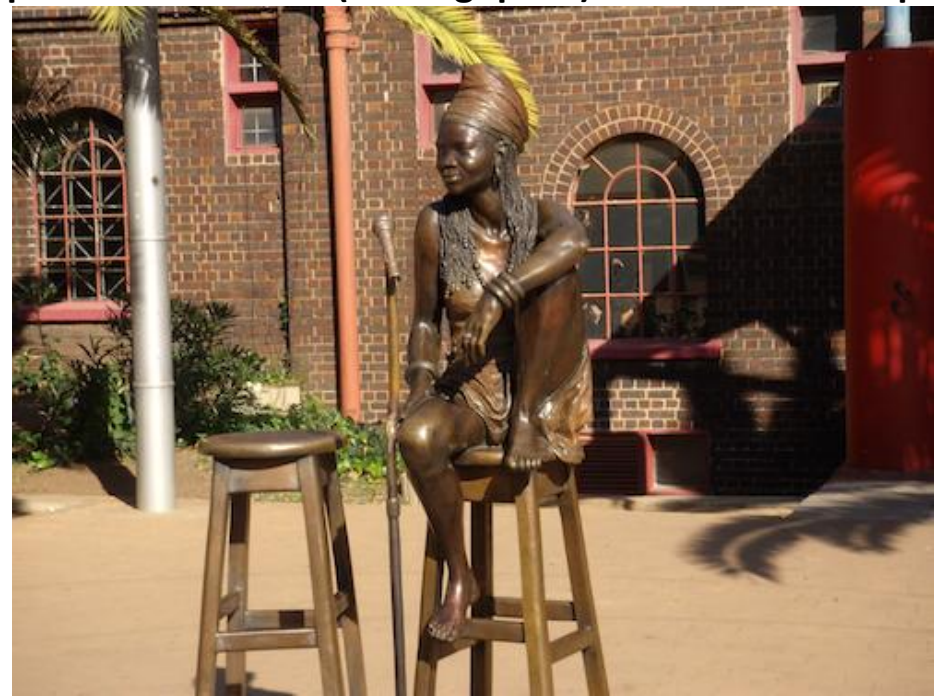

Cliché : P. Gervais-Lambony, 2007.

PG : Si l'on considère la chanson, il me semble qu'elle peut être étudiée de diverses manières eu égard à la nostalgie : soit comme support, soit comme déclencheur de celle-ci.

PGL : Bien sûr. On peut la traiter de la même façon que la littérature, c'est-à-dire s'interroger sur le discours qui est produit (par qui ? pourquoi ?) et sur l'effet de ce discours. Et, je pense que la chanson populaire ou la littérature populaire a un effet plus vaste que... Marcel Proust ! Et encore, cela se discute. Les chansons, en tous cas, irriguent nos vies, et pas seulement les chansons contemporaines. Si vous écoutez la célèbre radio qui s'appelle Nostalgie, vous vous s'apercevrez que les auditeurs qui appellent pour poser des questions ne sont pas si vieux qu'on pourrait le penser, en tout cas pas seulement! Donc oui, la chanson populaire suscite de la nostalgie et, en même temps, le souvenir des chansons de son passé sont support de la nostalgie. Je ne suis compétent que pour travailler sur les paroles des chansons, mais on peut pour aller plus loin se référer au Paysage des chansons de Renaud de Johanna Copans (2014). Elle propose, à la suite d'autres, une étude globale de la chanson, la " cantologie ", c'est-à-dire une approche qui prend en compte tout à la fois les textes des paroles, la musique, la personnalité du chanteur et les mises en scène des concerts. C'est un livre formidable. II faut faire attention au titre : le Paysage de la chanson de Renaud, c'est-à-dire qu'il s'agit d'analyser la production par un chanteur d'un paysage. J. Copans montre bien que Renaud s'inspire à l'origine de la chanson populaire française, avant de s'intéresser à la banlieue contemporaine, mais qu'il est aussi inspiré par des espaces, des lieux, dont il transmet une image forte qui est largement diffusée. Il y a beaucoup de références à la géographie dans cet ouvrage et en géographe on pourrait faire beaucoup de choses dans ce champ parce qu'il y a beaucoup de chanteurs qui se revendiquent d'un type d'espace et diffusent en même temps un discours sur ces espaces. Bruce Springteen en est un remarquable exemple : il est le chanteur des milieux populaires états-uniens et des espaces en cours de désindustrialisation, des espaces perdus et oubliés ; il est le chanteur des transformations urbaines et bien souvent de la nostalgie. 
PG : Permettez-moi maintenant de vous poser un question un peu provocatrice : faut-il être nostalgique pour travailler sur la nostalgie ?

PGL : Votre question est plus large en réalité, non ? II s'agit de se demander ce qu'on fait de sa propre émotion dans sa recherche. Alors, je pense qu'il faut en faire quelque chose. Je pense même qu'une bonne recherche ne peut être que liée à des émotions, précisément parce que c'est ce qui met en mouvement. Et, c'est vrai de n'importe quelle émotion : une révolte ou un sentiment d'injustice par exemple. Amartya Sen évoque cela quand il dit qu'il a commencé à travailler sur la justice [voir notamment: Sen, 2012] parce qu'il a éprouvé un sentiment d'injustice, donc a été mis en mouvement par une émotion. Mais cela vaut aussi pour un sentiment amoureux (pour une personne ou un lieu), pour un sentiment nostalgique, etc. Dans mon cas particulier, le fait d'éprouver de la nostalgie est lié à une durée passée sur un terrain, et c'est le cas chez beaucoup de gens. Si l'on travaille pendant longtemps sur un terrain, on le voit changer et, à un moment, on se retrouve dans une situation qui est assez proche de celle de quelqu'un qui a habité ce lieu pendant longtemps. Dans le cas de l'Afrique du Sud, c'est particulièrement fort. J'ai commencé à y travailler au début des années 1990. Or, l'espoir, l'optimisme, l'enthousiasme de cette époque-là ont progressivement diminué jusqu'à être remplacés par de l'inquiétude ou de la déception. On a la nostalgie des sentiments du passé aussi. Donc oui, je pense qu'il faut être nostalgique pour travailler sur la nostalgie. De même que je pense qu'il faut être révolté par des injustices pour travailler sur des injustices. Sinon, à mon avis, cela n'a pas de sens.

PG : Cela me fait penser à l'article de l'anthropologue Judith Hayem (2013) dans lequel elle revient sur les raisons de son amour puis de son désamour pour son terrain sud-africain. Mais, chez les géographes, notamment français, il semble y avoir une espèce de non-dit à ce sujet. Ces derniers parlent rarement de leurs émotions, du moins dans leurs écrits. Or comme vous le disiez justement, nos terrains, nos sujets de recherche partent de nos émotions. Comment expliquez-vous ce silence ? Pensez-vous que cette question mérite d’être formalisée ?

PGL : Absolument. A ce sujet, nous avions d'ailleurs organisé avec J. Hayem une table ronde lors d'un colloque africaniste à Bordeaux en $2010^{7}$. Il s'agissait d'inviter des gens qui avaient une pratique de terrain et de les interroger sur leurs éventuelles relations affectives, amoureuses à ce terrain. Indirectement, l'inspirateur de ce projet, était Roland Barthes (1977) : I'idée était de se demander si l'on pouvait ressentir les sentiments amoureux qu'il analyse dans une relation non pas à une personne mais à un terrain. Et je pense que oui : comment bien travailler sur un terrain que l'on aime pas ? Cela arrive! J'ai vu des doctorants qui n'arrivaient plus à avancer parce qu'ils n'aimaient pas leur terrain, non pas parce qu'ils n'aimaient pas faire du terrain, mais parce qu'ils n'aimaient pas ce terrain-là. Ils étaient profondément déçus par rapport à ce qu'ils avaient imaginé y rencontrer. Je viens d'une tradition de géographie revendiquant l'objectivité, la prise de distance, mais il faut que cela évolue. Si l'on reste trop extérieur dans son discours, on occulte les effets de sa positionnalité, qui existent toujours. D'ailleurs, si l'on considère que nos méthodologies visent à garantir l'objectivité de nos résultats, n'y a-t-il pas une contradiction avec une recherche qui se reconnaît pour une part au moins non-objective ? Dès lors, la seule

\footnotetext{
${ }^{7}$ Voir : http://etudes-africaines.cnrs.fr/atelier/glissement-de-terrain-atelier-24-glissements-amoureux/
} 
manière de parler des émotions - j'ai conscience que c'est un peu extrémiste - ce serait sans doute de les éprouver.

Pour moi, l'un des plus beaux textes de géographie qu'on a jamais écrit et qu'on écrira peut-être jamais sur l'Afrique du Sud, c'est Noirs et Blancs de Jacques Weulersse (1931). Ce texte rentre mal dans la catégorisation de texte scientifique. C'est un récit de voyage mais dans lequel l'auteur exprime ses sentiments, et, en même temps, c'est un magnifique texte de géographie. C'est remarquable comme source d'informations et par les sentiments, les prises de position que J. Weulersse, en tant qu'anti-ségrégationniste et anti-colonialiste, nous transmet, et qui nous permettent que comprendre plus largement l'Afrique du Sud. II raconte les lieux en mobilisant tout ce que d'habitude, en tant que chercheur, on se croit obligé d'effacer au motif que cela relève du registre de l'anecdotique, du personnel, du subjectif. Souvent, dans des thèses de doctorats par exemple, vous trouvez cela quelque part en début d'introduction, dans les remerciements ou dans des lieux un peu annexes, comme si cela ne devait pas rentrer ou n'avait pas sa place dans le texte. Je pense qu'au contraire il faut donner sa place à cela, mais sans tomber dans l'excès inverse qui consisterait à en faire l'objet principal du discours, à ne parler plus que de son sentiment et de sa propre relation au terrain. C'est d'ailleurs ce que nous apprennent ce qu'on appelle les études post-coloniales : il est essentiel de dire d'où l'on parle, qui l'on est et quelles relations on a avec son objet ou son terrain.

Et cela vaut même pour des émotions négatives, telles que la peur. Je pense à l'Afrique du Sud bien sûr, parce qu'on partage ce type d'émotions avec des gens sur le terrain. A ce sujet, il y a aussi toute une réflexion à mener sur des formes de co-production du travail scientifique de terrain en tant qu'il implique des relations humaines, des sentiments qui ont des effets.

\section{Inscription dans le champ disciplinaire : nostalgie et géographie}

PG : Venons en à présent à des questions sur l'inscription dans le champ disciplinaire des émotions. A votre avis, quelle peut être la place des émotions en géographie (française) ?

PGL: Honnêtement, je pense que la géographie, c'est ce que font les géographes, c'est-à-dire des gens qui ont envie de se revendiquer, de se définir de cette façon. Et cela a toujours été le cas. Par ailleurs, on a toujours sous-estimé le rôle des émotions chez nos prédécesseurs. Qu'on ne me dise pas que la géographie d'Yves Lacoste, par exemple, et plus largement la géopolitique, ne sont pas liées à une émotion qui est de l'ordre d'une révolte, d'un sentiment d'une nécessité de protester. Et cela a donné lieu à bien des débats en géographie. Y. Lacoste que je l'ai eu comme enseignant en DEA - nous disait : vous devez avoir un positionnement, un engagement politique, sinon ce que vous ferez sera sans intérêt. C'est un vrai point de débat, bien sûr, mais il est certain que cela a été d'une grande importance pour moi.

Si l'on pense à des auteurs plus anciens, comme Pierre George ou Jean Dresch, on a également affaire à des chercheurs qui avaient des engagements politiques très forts. Pourtant du fait de la séparation stricte entre science et subjectivité, tout se passait, en apparence, comme s'ils avaient deux vies parallèles. Mais on sait bien pourtant que ces deux vies, de géographe et d'engagement, s'influençaient fortement l'une l'autre. L'expérience pour Jean Dresch des 
terrains de l'époque coloniale en Afrique du Nord a eu des effets sur son positionnement politique et, à l'inverse, il a écrit des choses liées à son engagement politique. C'est une évidence aussi pour Pierre George, avec des évolutions et des changements notables au cours du temps. Et puis, si l'on pense à Gilles Sautter, pour citer quelqu'un qui m'est plus proche parce que c'était mon maître avant la thèse, c'est quelqu'un qui a toujours exprimé un intérêt pour les sentiments humains et pour les formes d'expressions artistiques. Cela se voit dans ses écrits les plus tardifs, comme s'il avait pu évoquer cet intérêt à un moment où il était libéré de la contrainte de tenir un discours strictement scientifique ou strictement " de géographie ». Quant à Michel Coquery, il avait trouvé sa solution personnelle en étant géographe sur l'urbain d'un côté, et artiste de l'autre, mais ces deux facettes étaient très liées. Il s'agissait souvent dans ses collages de dire autrement sa fascination en même temps que son inquiétude sur le monde des villes. Donc ce désir et ce besoin d'exprimer ses sentiments existent depuis longtemps. Mais c'est une bonne chose si aujourd'hui on est plus à l'aise et plus libre vis-à-vis de cela.

Mais il y a autre chose, au-delà de la question des émotions des chercheurs eux-mêmes et qui est peut-être plus important. Si la géographie, ou une partie de la géographie, est une science humaine, elle cherche à rendre compte de l'expérience humaine de l'espace, or celle-ci est influencée par les sentiments et les émotions. Impossible dès lors de ne pas les étudier. Les étudier c'est faire une " géographie de l'expérience » (Tuan, 1971), je crois que c'est important, et... utile. Le risque sinon est assez clair pour la discipline il me semble : laisser de côté tout un pan de ce qu'est l'espace dans lequel vivent réellement les humains. Est-ce que cela ne serait pas dommage?

\section{Retour à Comet}

PG : Pour finir, j'aimerais que nous retournions à Comet et que nous nous arrêtions sur cette photographie (Figure 3).

Figure 3 : L’église de Comet, un tomason ?

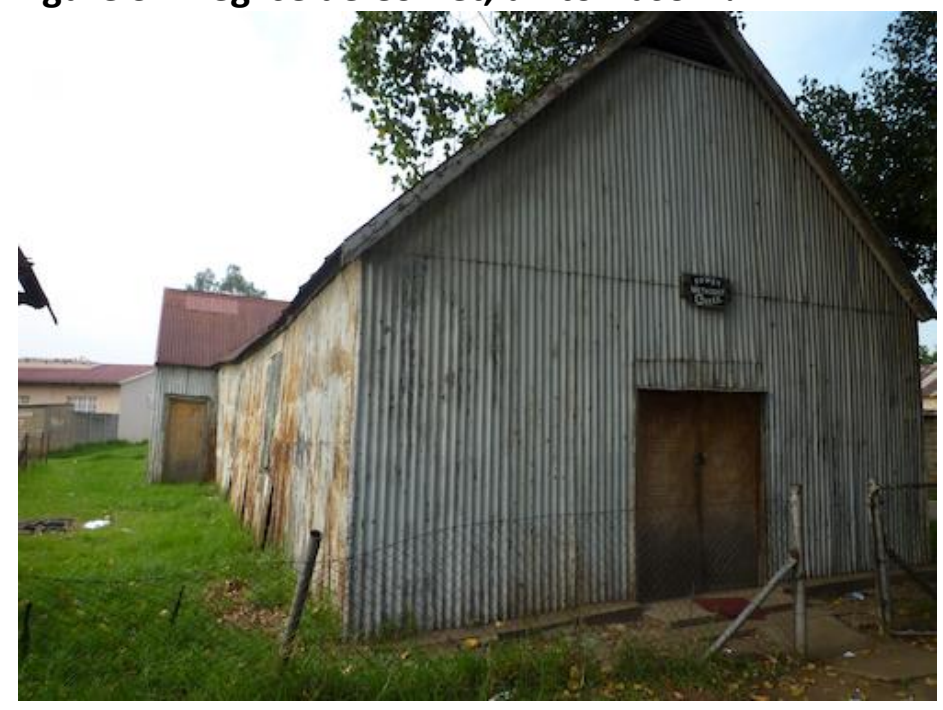

Cliché : P. Gervais-Lambony, 2011. 
PGL : Si cette photographie est importante c'est parce que c'est une église (comme la " petite église " de Ménilmontant chantée par Trenet). II me semble que quand on parle d'émotions et de sentiments, l'interrogation sur le religieux est à prendre en compte. Le bâtiment religieux dans beaucoup de quartiers, et en Afrique du Sud c'est particulièrement vrai, reste un symbole (et le plus souvent, quand les autorités urbaines de l'apartheid rasaient un quartier elles laissaient en place les lieux de cultes). Le religieux, la religiosité, ce sont des sentiments, non ? Peu de géographes ont travaillé sur le religieux en tant que spiritualité depuis Pierre Deffontaines (1948), cela ne devrait pas être un sujet tabou à mon avis. Cette église est complètement désaffectée aujourd'hui, mais personne ne l'a squatté, personne ne l'habite, personne non plus n'a pensé à la détruire. Elle n'est pourtant rien d'autre qu'une petite fenêtre sur un temps qui n'existe plus et que beaucoup de résidents du quartier n'ont d'ailleurs pas connu... ils peuvent seulement imaginer, et ce n'est pas rien !

PG : Un tomason en somme.

PGL : Oui.

PG : Sur ce tomason, il ne me reste plus qu'à vous remercier.

PGL : De rien!

\section{Bibliographie}

APPADURAI A. (2005 [1996]) Après le colonialisme: les conséquences culturelles de la globalisation, Paris, Payot \& Rivages.

AKASEGAWA G. (2010 [1987]) Hyperart: Thomasson, New York, Kaya Press.

BARTHES R. (1977) Fragments d'un discours amoureux, Paris, Seuil.

BOYM S. (2001) The future of nostalgia, New York, Basic Books.

CERTEAU M. de (1994 [1980]) L'Invention du quotidien (t.2) : Habiter, cuisiner, Paris, Gallimard.

COPANS J. (2014) Le paysage des chansons de Renaud, Paris, Editions L'Harmattan.

DAVIS M. (1997) City of quartz: Los Angeles, capitale du futur, Paris, Éd. la Découverte.

DEFFONTAINES P. (1948) Géographie et religions, Paris, Gallimard.

DLAMINI J. (2010) Native Nostalgia, Jacana Media.

DORIER-APPRILL E. et GERVAIS-LAMBONY P. (dir) (2007) Vies citadines, Paris, Belin.

GERVAIS-LAMBONY P. (2003) Territoires citadins : 4 villes africaines, Paris, Belin.

GERVAIS-LAMBONY P. (2012) "Nostalgies citadines en Afrique Sud » EspacesTemps.net [en ligne], [URL : http://www.espacestemps.net/document9459.html]

HARVEY D. (1989) The Condition of Postmodernity, Oxford, Cambridge, Blackwell.

HAYEM J. (2013), "Glissement amoureux et glissement politique dans le face-à-face avec la xénophobie " in Annie Benveniste (dire) Se faire violence, analyses des coulisses de la recherche, Paris, Tetraèdre, p. 63-86.

MPHAHLELE E. (1985 [1959]) Down Second Avenue, London, Boston, Farrar, Straus and Giroux. SEN A. (2012) L'idée de justice, Paris, Flammarion.

TUAN Y.-F. (1971) Space and place: The perspective of experience, Univ. of Minnesota Press.

VLADISLAVIC I. (2006) Portrait with keys, London, Portobello.

WEULERSSE J. (1931) Noirs et Blancs à travers l'Afrique nouvelle, Paris, A. Colin. 